\title{
Innate Cytokine Responses and Toll-Like Receptor Induced by Recombinant Porcine Rotavirus VP6 and VP7 Proteins Expressing in Lactobacillus plantarum NC8 Strain Colonization in Mice
}

\author{
Seria Masole Shonyela1,2*, Wentao Yang1, \\ Guilian Yang1, Chunfeng Wang ${ }^{1}$ \\ ${ }^{1}$ College of Animal Science and Technology, Jilin University, Changchun, China \\ ${ }^{2}$ Ministry of Agriculture, Livestock and Fisheries, Veterinary Complex, Dar es Salaam, Tanzania \\ Email: *seria.masole@sacidas.org
}

How to cite this paper: Shonyela, S.M., Yang, W.T., Yang, G.L. and Wang, C.F. (2020) Innate Cytokine Responses and Toll-Like Receptor Induced by Recombinant Porcine Rotavirus VP6 and VP7 Proteins Expressing in Lactobacillus plantarum NC8 Strain Colonization in Mice. World Journal of Vaccines, 10, 17-31. https://doi.org/10.4236/wjv.2020.101002

Received: December 12, 2019

Accepted: January 14, 2020

Published: January 17, 2020

Copyright (อ 2020 by author(s) and Scientific Research Publishing Inc. This work is licensed under the Creative Commons Attribution International License (CC BY 4.0).

http://creativecommons.org/licenses/by/4.0/

\section{(c) (i) Open Access}

\begin{abstract}
The significant function of Toll-like receptors (TLR) is the detection of microbes by host guard cells that guide to the innate immune responses and to the successive adaptive. The current study patterns of TLR2, TLR3 and TLR9 expressing antigen presenting cells (APCs) in blood of mice after colonization with $L$. plantarum NC8 strain were assessed. The power of $L$. plantarum on serum innate cytokine and TLR responses stimulated by recombinant NC8pSIP409-pgsA-VP6-DCpep, NC8-pSIP409-pgsA-VP7-DCpep and NC8-pSIP409pgsA were also assessed. We confirmed that $L$. plantarum NC8 stimulated powerful TLR2 expressing APC responses in blood Recombinant strain stimulated a TLR3 response in spleen, and TLR9 responses were stimulated in blood or in spleen. Recombinant NC8-pSIP409-pgsA-VP6-DCpep, NC8pSIP409-pgsA-VP7-DCpep on TLR2 and TLR9 expressing APC responses has a preservative outcome, reliable with the DCpep adjuvant outcome. In serum the recombinant NC8-pSIP409-pgsA-VP6-DCpep, NC8-pSIP409-pgsAVP7-DCpep has increased the IL-4 and IFN- $\gamma$ responses, except that on the TLR3 and TLR9 expressing CD14 APC responses it had an oppressive consequence in spleen and the IFN- $\alpha$ response in serum-stimulated by PRV. Our results give details that following PRV infection after immunization with NC8-pSIP409-pgsA-VP6-DCpep, NC8-pSIP409-pgsA-VP7-DCpep, the systemic TLR2, TLR3, and TLR9 expressing CDC and macrophage/monocyte responses.
\end{abstract}




\section{Keywords}

Toll-Like Receptors, Recombinant, Porcine Rotavirus, Lactobacillus plantaram, Mice

\section{Introduction}

The significant function of Toll like receptor (TLRs), as kind of pattern-recognition receptor (PRR), is connection inborn and adaptive immune and inborn immunity responses and viral antigen identification [1] [2] [3]. On many cell types the TLRs can be expressed e.g. macrophages/monocytes and DCs. Through several signaling pathways the toll like receptor activation induces type I interferon production that leads to anti-viral and proinflammatory cytokine responses and induction of adaptive immune responses [4] [5] [6]. The recognition of different microbe-associated molecular patterns (MAMPs) is one of the roles of different TLRs [5]. The recognition of lipoteichoic acids, peptidoglycans and lipopeptides from bacteria is the role of TLR1in association with TLR2 and TLR6 [7] [8]. Toll like receptor 3 recognizes dsRNA, which is found many viruses like in rotavirus genome during their replication cycles. For worldwide the mainly vital etiologic agent of severe gastroenteritis in young animal and young children is rotaviruses [9]. Therefore, in systemic lymphoid tissues the TLR may contribute to the anti-viral immunity.

In animals and humans Lactic acid bacteria, including lactobacilli are broadly appraised as probiotics [10] [11] and have been shown in young animals and have been shown to significantly stimulate gut epithelial cell proliferation enhance innate and acquired immunity in young animals and children and suppress intestinal inflammation [12] [13] [14]. The severity of acute rotavirus gastroenteritis in children has been shown to reduce by different LAB strains [15] [16]. In the current study we focused on the effect of L. plantarum NC8 on adaptive and innate immune responses to porcine rotavirus infection and the effect of DCpep adjuvant on porcine rotavirus vaccines. The effect of different adjuvants of several LAB strains has been reported in pigs and in humans [17] [18] [19] [20] [21]. However, the mechanism is undefined.

Our study focused on the effect of recombinant $L$. Plantarum NC8 expressing VP6 and VP7 on early cytokine responses as via pattern recognition receptor (PRR); they are indicators of innate immune cells activation and function in the innate immune response. It directly interacts with intracellular TLR3 and enhances dsRNA-mediated TLR3 activation by supporting uptake of dsRNA into cells [22]. In this study to elucidate the effect of $L$. Plantarum NC8 on different APC in systemic lymphoid tissues the influences of LAB on frequencies of TLR2, TLR3 and TLR9 expressing CD14+ versus CD14- DCs and macrophages/monocytes in the blood and spleen of PRV infected mice were evaluated. Thus, the understanding of treatment and prevention of viral diseases in animals and humans on how 
TLR3 is regulated and activated immune cells can help to select efficient therapies.

\section{Materials and Methods}

\subsection{Chemical and Enzyme}

All chemicals for cloning, expression, and purification of recombinants, were purchased from Bioss China and provided by Laboratory of Jilin Provincial Engineering Research Center of Animal Probiotics, in Jilin Agricultural University of China.

\subsection{Mice}

In this model, we used Rotavirus antibody-free adult Female Balb/C mice 7 weeks of age (weighing 25-30 g) obtained from Beijing Huafukang Biotechnology Co., Ltd. China used for the immune responses to recombinant $L$. plantaram NC8-pSIP409-pgsA-VP6-DCpep or L. plantaram NC8-pSIP409-pgsA-VP7-DCpep strain. This study was carried out in agreement with the principles established by Jilin Agriculture University Changchun China and guide for the use of laboratory and care animals and all experimental protocols were approved by a Jilin Agriculture University. Sixty (60) mice were randomly divided into four groups and were housed under standard conditions with free access to food and water.

\subsection{Strains and Culture Conditions}

Porcine rotavirus isolate DN30209 VP7 protein mRNA, complete cds (GenBank: JN388691.1) and VP6 gene, complete cds (GenBank: JN977137.1) were kindly purchased from genewiz.com and provided by Laboratory of Jilin Provincial Engineering Research Center of Animal Probiotics. Bacteria strain, plamids and virus were kindly provided by Laboratory of Jilin Provincial Engineering Research Center of Animal Probiotics. L. plantarum was grown anaerobically in MRS broth at $37^{\circ} \mathrm{C}$ without shaking in plate on MRS medium with $1.5 \%$ agar at $37^{\circ} \mathrm{C}$ erythromycin at $10 \mu \mathrm{g} / \mathrm{ml}$ final concentration were added where appropriate to the culture medium and growth were monitored for $12 \mathrm{~h}$.Then single colony were grown in MRS liquid medium, In case of interventions with L. plantarum, by centrifugation the cells were harvested and three times wash with a physiological salt solution (buffer solutions) Viruses and Cell Culture.

\subsection{Expression Plasmid Construction}

\section{Polymerase chain reaction (PCR) of VP6 gene proteins}

Porcine rotavirus isolate DN30209 VP6 gene, complete cds (GenBank: JN977137.1) was amplified by polymerase chain reaction (PCR) Specific primers were designed by Primers premier 5.0. The PCR reaction was carried out in standard $50 \mu \mathrm{l}$ reaction for amplification of VP6 genes using the primer sets following standard protocols. The tubes were then placed in a thermocycler and PCR reaction conditions for porcine rotavirus VP6 gene were standardized with 
initial denaturation step of $98^{\circ} \mathrm{C}$ for 5 min followed by 30 cycles of $98^{\circ} \mathrm{C}$ for 0.01 $\min , 55^{\circ} \mathrm{C}$ for $30 \mathrm{sec} \min , 72^{\circ} \mathrm{C}$ for $30 \mathrm{sec}$ and final extension of $72^{\circ} \mathrm{C}$ for $4 \mathrm{~min}$ [23]. Porcine rotavirus isolate DN30209 VP7 protein mRNA, complete cds (GenBank: JN388691.1) was cut by double enzyme Hind III and Xba I from T4-VP7DCpep plasmid.

Electroporation of $L$. plantarum was carried out as follows: $10 \mu \mathrm{l}$ of plasmid was gently added to $100 \mu \mathrm{l}$ of $L$. plantarum NC8, and mixed gently for $5 \mathrm{~min}$ at $4^{\circ} \mathrm{C}$ and were subjected to an electric pulse. The mixture of plasmids DNA and L. plantarum NC8 were then anaerobically without E.M incubated in MRS medium for $2 \mathrm{~h}$ at $37^{\circ} \mathrm{C}$. Recombinant NC8-pSIP409-pgsA-VP6-Dcpep and NC8pSIP409-pgsA-VP7-Dcpep were picked on MRS agar medium including E.M. The transformants of $L$. plantarum NC8 sequences were verified by plasmid DNA sequencing.

\subsection{Immunization of Recombinant L. plantarum Strainsto BALB/c Mice}

Mice were orally immunized with $109 \mathrm{CFU}$ of $L$. plantaram strains and the immune protocol was administered on three consecutive days: the inoculation at days 0,1 , and 2; a booster at days 14,15, and 16; a second booster at days 28, 29, and 30. Mice sera were collected on days $0,9,25$, and 41 following the first immunization to evaluate the immunogenicity of the recombinant $L$. plantarum NC8. PBS was given in the similar approach to the control groups.

\subsection{Sample Collection from Immunized Mice}

To isolate mononuclear cells (MNCs) from spleen and peripheral blood; the blood, and fecal pellets were collected from mice 14 days after the first and second booosting immunization of NC8-pSIP409-pgsA-VP6-DCpep or NC8pSIP409-pgsA-VP7-DCpep. On the same day of MNC isolation the isolated MNCs were stained with antibodies to mice cell markers and TLR antibodies straight without in vitro stimulation.

\subsubsection{Enumeration and Recording of LAB}

All samples (fecal dilution washes) were diluted in $4 \mathrm{ml}$ of peptone water $0.1 \%$ then plate onto MRS agar. The plates were incubated in sealed anaerobic jars at $37^{\circ} \mathrm{C}$ for $24 \mathrm{hrs}$. Then we recorded and enumerated the number of CFU on plates. Shedding of LAB was expressed as CFU/ml. To assess the Bacteremia the mice serum plate onto MRS agar plates and incubated in sealed anaerobic jars at $37^{\circ} \mathrm{C}$ for $24 \mathrm{hrs}$.

\subsubsection{Flow Cytometry Analysis}

An antibody to porcine $\mathrm{CDC}$ and macrophage/monocyte markers was first used to stain the MNCs, followed with an antibodies to TLR2, TLR3 and TLR9. At each step MNCs were once washed and incubated at room temperature for 15 min. First cells was stained with mouse anti-porcine VP6 IgG, and mouse an- 
ti-porcine VP7 IgG. After washing the secondary fluorescent conjugated antibodies, fluorescein isothiocyanate conjugated and allophycocyanin conjugated rabbit anti-porcine IgG, was added. The cells washed twice, and then added the biotin conjugated rabbit anti porcine IgG1, then streptavidin conjugated with peridinine chlorophyll protein were added. The PE conjugated mouse IgG1 isotype control and phycoerythrin conjugated mouse anti-porcine TLR2 mouse IgG1 was added for staining of TLR2, which is expressed on the cell surface, the TLR2 and its alike isotype match control tubes. Before flow cytometry analysis the cells were incubated, washed three times, fixed and resuspended in staining buffer and kept at $4^{\circ} \mathrm{C}$ in dark. By using a 4 color FACSCalibur flow cytometer the analysis of the stained cells was performed and a minimum 22,000 cells were obtained. Data analysis was performing using FlowJo 7.6.1 software.

\subsubsection{ELISA Analysis for Detection of Serum Cytokine Levels}

Blood samples from mice were collected from the eye. Sera was processed and stored at $-20^{\circ} \mathrm{C}$ until tested. The ELISA was conducted using anti-mouse cytokine antibodies for detection of porcine IFN- $\gamma$, IL-4, IL-6, IL-10, IL-12, IFN- $\gamma$, TGF- $\beta$, and TNF- $\alpha$ as previously described [24].

\subsection{Statistical Analysis}

To evaluate frequencies of TLR-expressing cells in subpopulation of cells in spleen and blood among groups and the serum cytokine concentrations among groups the non-parametric Kruskal Wallis rank sum test was performed. The similar test was used when dissimilarities among these groups were detected, in a pairwise approach to make clear the nature of the dissimilarities. For the evaluation of association between frequencies of TLR-expressing macrophages/monocytes or cDCs and concentrations of cytokine in serum the Spearman's rank correlation test was used. At $p<0.05$ was judged as statistical significance throughout.

\section{Results}

\subsection{Fecal Counts of Lactobacillus plantaram, PRV Infection and Clinical Signs}

The mean LAB count in the recombinant $L$. plantaram NC8-pSIP409-pgsA-VP6DCpep or NC8-pSIP409-pgsA-VP7-DCpep group was significantly higher than the NC8-pSIP409-pgsA group on day 35. In the intestinal contents the bacterial strains of the recombinant NC8-pSIP409-pgsA-VP6-DCpep or NC8-pSIP409pgsA-VP7-DCpep fed BALB/c mice were evaluated by serially diluted plating samples on Lactobacillus selective LB agar plates and anaerobically cultured over night at $37^{\circ} \mathrm{C}$. There were identical morphology of the bacterial colonies on LB agar plates from the intestinal content and the colonies from Lactobacillus inoculum of the original. We also plated the daily rectal swab samples and serially diluted intestinal contents on blood agar plates and cultured aerobically over night at $37^{\circ} \mathrm{C}$. On the blood agar plates from any of the BALB/c mice, no bac- 
terial growth was detected which confirming that no irrelevant bacterial contamination happened.

In this study the PRV infection was confirmed by ELISA for the detection of PRV titers in intestinal contents from all the mice. The entire control group developed antigenemia, but none of the NC8-pSIP409-pgsA-VP6-DCpep or NC8-pSIP409-pgsA-VP7-DCpep mice developed bacteremia. The duration and severity of diarrhea between the NC8-pSIP409-pgsA-VP6-DCpep and NC8pSIP409-pgsA-VP7-DCpep and NC8-pSIP409-pgsA groups did not significantly differ therefore reduction of rotavirus diarrhea did not cause by intestinal colonization with the mixture of L. plantaram NC8.

\subsection{Flow Cytometry Analysis for Detection of TLR Expression in Mice by Using Anti-Porcine TLR Antibodies}

By flow cytometry the specificity and cross reactivity of anti porcine TLR2, TLR3 and TLR9 antibodies were assessed for detection of mice TLRs in MNCs. Adequate cross reactivity among mice TLR antibodies was indicated by the powerful fluorescent intensity and the specificity was indicated by the obvious partition of the histograms of isotype matched unrelated control antibody stained MNCs from the TLR antibody stained MNC (Figure 1). By the elevated amino acid sequence homologies among porcine and mice TLRs can explain the cross reactivity of the antibodies.

Using normal conventional mouse blood MNCs the sensitivity of the anti mouse TLR polyclonal antibodies were compared with the anti porcine TLR antibodies. Before staining of the MNCs were stimulated in vitro with peptidoglycan. Comparable frequencies of TRL9+ and TLR2+ monocytes were detected by anti mouse TLR antibodies and anti porcine antibodies (Figure 1). The frequencies of TLR2+ or TRL9+ detected by anti-mouse and anti-porcine antibodies the differences were not statistically significant and were less than $0.4 \%$. Due to our study results verified that the anti pig TLR antibodies are appropriate for study of TLR responses in pigs.

Mice were immunized with NC8-pSIP409-pgsA-VP6-DCpep or NC8-pSIP409pgsA-VP7-DCpep; NC8-pSIP409-pgsA. Macrophages/monocytes were defined as CD14+/-, +CD11R1-, CD14/TLR3.

Detection of frequencies of TLR-expressing cDCs and monocytes/macrophages in the three treatment groups is depicted by the representative dot plots in Figure 2 using TLR3 in spleen. The same has been described in a previous publication [17] [18] [19] [20] [21] that the tissue distribution and frequencies of mouse macrophages/monocytes and cDCs in the three groups. As shown in Figure 2 TLR3 had zero percent TLR3+ cells for the MNCs stained with the isotype matched irrelevant control antibody and antibodies to cell markers, this verifying the the antibody specificity. The frequencies mean of TLR2, TLR3 and TLR9 expressing CD14+ and CD14- macrophages/monocytes and cDCs in general, elevated detection of frequencies of TLR expressing CD14+ APCs than CD14- APCs. 
Within the treatment groups in both spleen and blood high unpredictability was observed for frequencies of TLR expressing APCs.

\subsection{TLR Expressing APCs in Spleen}

\subsubsection{Induction of TLR2 Expressing CD14+ APCs by Either NC8 or PRV}

Detection of TLR expressing macrophages/monocytes or cDCs in spleen of control mice were minimal frequencies. But $L$. plantaram NC8-pSIP409-pgsA-Vp6-DCpep or L. plantaram NC8-pSIP409-pgsA-Vp7-DCpep; significantly higher frequencies were induced of CD14+ TLR2 expressing macrophages/monocytes and cDCs which representing the contribution of TLR2 in identification of PRV infection

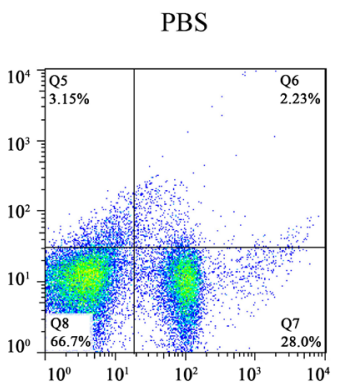

NC8-pSIP409-pgsA

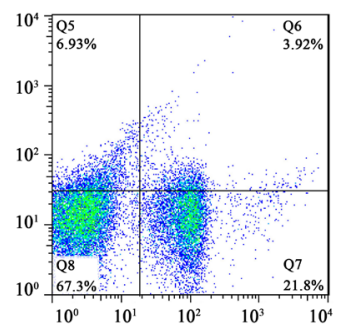

NC8-pSIP409-pgsA-VP6-DCpep

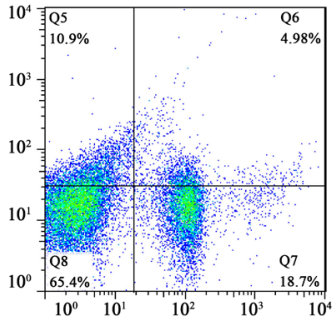

NC8-pSIP409-pgsA-VP6-VP7-DCpep

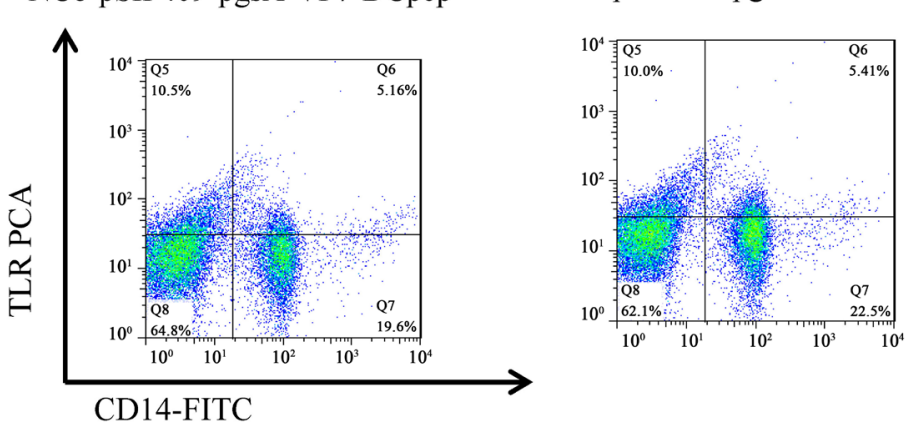

CD14-FITC

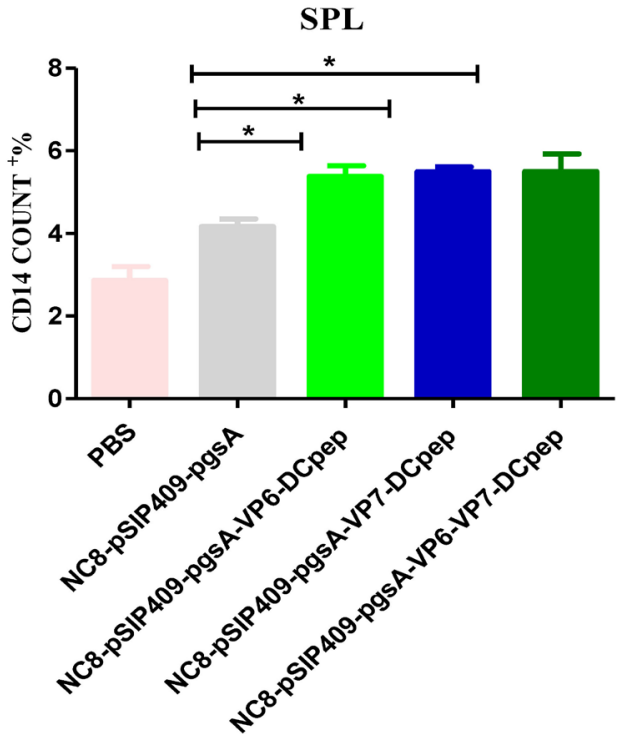

Figure 1. Frequencies of TLR3-expressing splenic monocytes/macrophages of mice from each treatment group. 


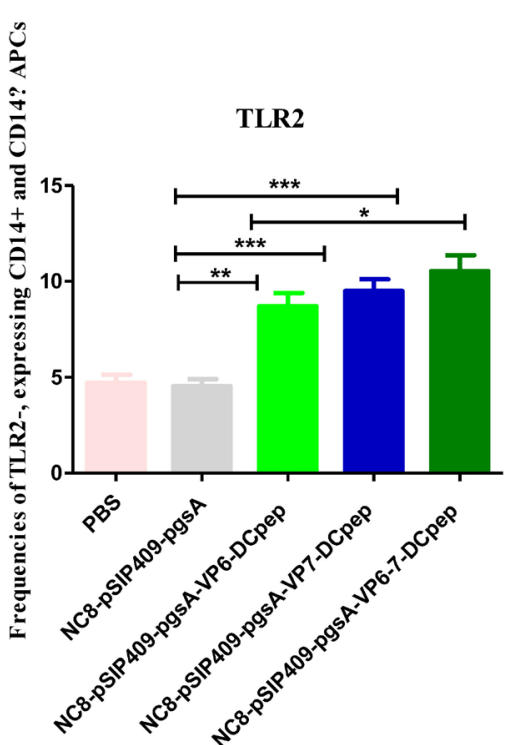

Frequencies of TLR2-expressing CD14+ and CD14- APCs in spleen of $\mathrm{BALB} / \mathrm{c}$ mice

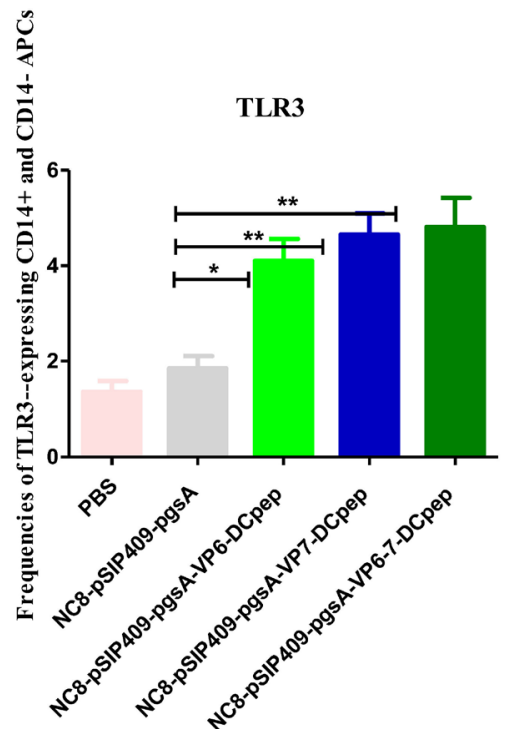

Frequencies of TLR3-expressing CD14+ and CD14- APCs in spleen of $\mathrm{BAI}, \mathrm{B} / \mathrm{c}$ mice

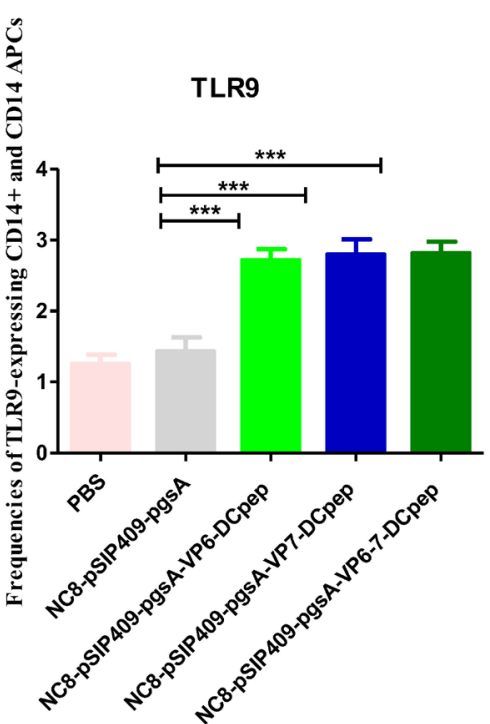

Frequencies of TLR9-expressing CD14+ and CD14- APCs in spleen of BALB/c mice

Figure 2. Frequencies of TLR2-, TLR3-, and TLR9-expressing CD14+ and CD14- APCs in spleen of BALB/c mice.

and L. plantaram NC8 colonization. Except for the low TLR2 expression on cDCs in the NC8-pSIP409-pgsA group, on the contrary to the CD14+ APCs, CD14- macrophages/monocytes and CDCs had minimum TLR2 expression.

\subsubsection{Induction of TLR3 Expressing CD14+ and CD14- APCs by PRV While on Frequencies of TLR3-Expressing CD14- APCs the NC8 Had an Antagonistic Effect with PRV}

There was significantly elevated frequencies induction of TLR3 expressing CD14+ APCs in L. plantaram NC8-pSIP409-pgsA-Vp6-DCpep or L. plantaram NC8-pSIP409-pgsA-Vp7-DCpep groups than controls mice, and they were comparable to the NC8-pSIP409-pgsA group. On the contrary, there were significantly lower frequencies of TLR3 expressing CD14- macropahges/monocytes and cDCs in the L. plantaram NC8-pSIP409-pgsA-Vp6-DCpep or L. plantaram NC8pSIP409-pgsA-Vp7-DCpep group than frequencies in the control group, signifying an adjuvant DCpep antagonistic effect on the PRV stimulated TLR3 response. Minimal frequencies induced by NC8-pSIP409-pgsA of TLR3 expressing CD14- cDCs and CD14+ in spleen, matched with the low cytokine responses

(Figure 3). This result was consistent with some previous study by [25].

\subsubsection{Induction of TLR9 Expressing CD14+ and CD14- APCs by Recombinants}

The TLR9 expressing CD14+ and CD14- APCs patterns in the L. plantaram NC8-pSIP409-pgsA-Vp6-DCpep and L. plantaram NC8-pSIP409-pgsA-Vp7-DCpep groups were comparable to patterns of TLR3 with somewhat lesser frequencies as shown in (Figure 2), signifying that TLR9 is as well engaged in the PRV innate immune responses induction. There was minimal induction of TLR9 expressing 

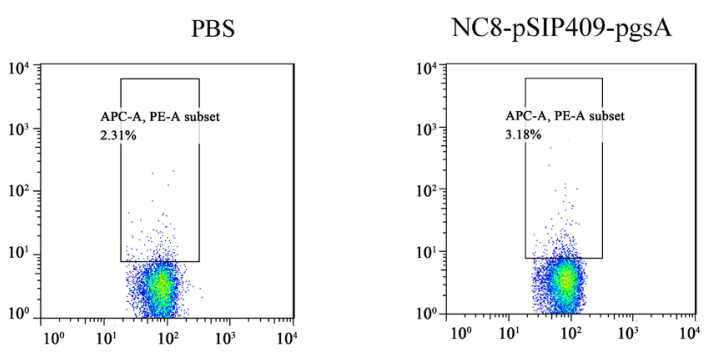

NC8-pSIP409-pgsA-VP6- DCpep
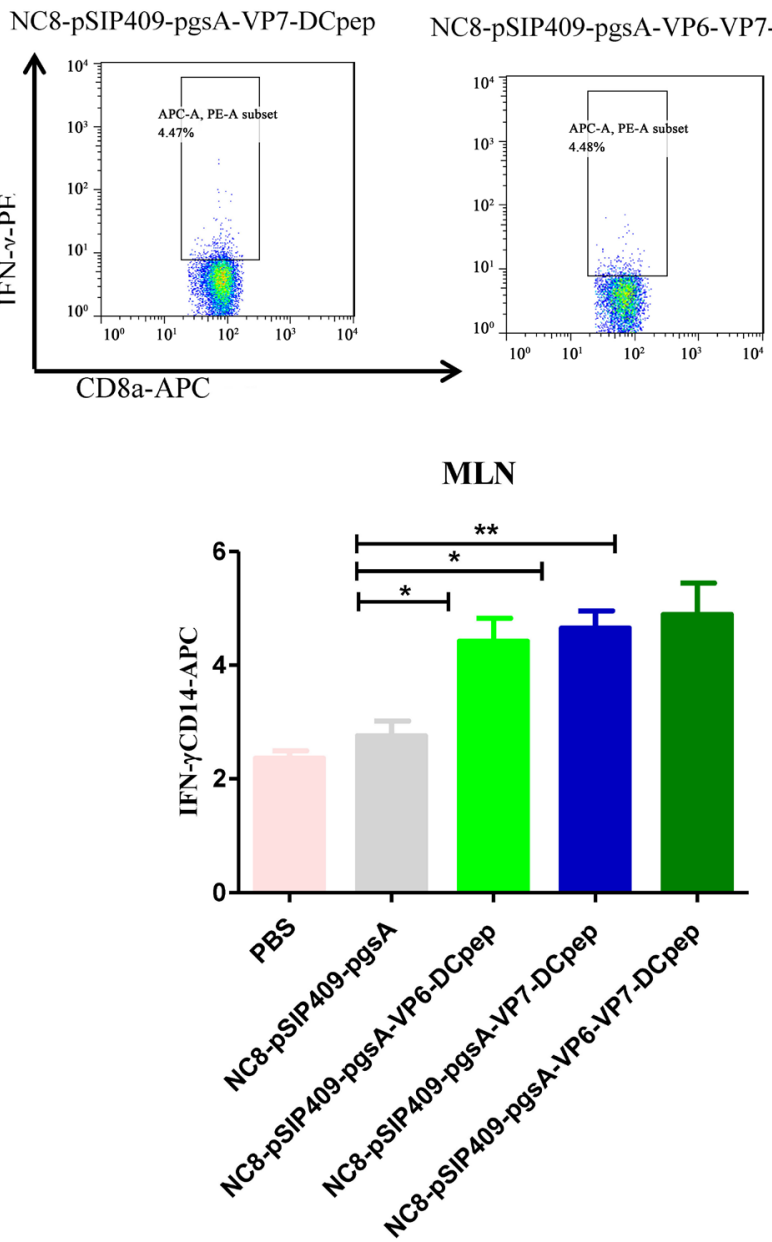

The expression of $\mathrm{CD}^{+} 4^{+} \mathrm{IFN}-\boldsymbol{\gamma}^{+} \mathrm{T}$ cells in mouse MLN

Figure 3. Cytokine levels in serum and the correlation with TLR responses.

CD14+ and CD14- APCs in spleen from the L. plantaram NC8-pSIP409-pgsA group.

\subsection{TLR-Expressing APCs in Blood}

\subsubsection{High Frequencies Induction of TLR2 Expressing CD14+ APCs by Recombinant Strains}

Blood from PBS inoculated mice group minimal to no TLR expressing CD14+ or CD14- macrophages/monocytes were detected (Figure 2). Though, there was low detection of frequencies of TLR2- and TLR3 expressing CD14+ and CD14- 
cDCs, which probable mirror the constitutive baseline expression of the TLRs by blood cDCs. The highest mean frequencies of TLR2 expressing CD14+ cDCs induced by $L$. plantaram NC8-pSIP409-pgsA-Vp6-DCpep or L. plantaram NC8pSIP409-pgsA-Vp7-DCpep in blood contrasted to NC8-pSIP409-pgsA group and they were significantly higher than the PBS control group.

TLR9-expressing CD14+ APCs were induced by recombinant strains

The TLR9 expressing CD14+ APCs pattern was comparable to the TLR2 pattern, however at lower magnitude about 10 fold, with the two L. plantaram NC8-pSIP409-pgsA-VP6-DCpep or L. plantaram NC8-pSIP409-pgsA-VP7-DCpep groups significantly higher induction of frequencies of TLR9 expressing CD14+ cDCs contrasted to the NC8-pSIP409-pgsA and PBS groups. Our results point out that in blood mostly induced TLR2- and TLR9-expressing cDCs by L. plantaram NC8-pSIP409-pgsA-VP6-DCpep or L. plantaram NC8-pSIP409-pgsAVp7-DCpep had an preservative outcome on frequencies of TLR2 and TLR9 expressing cDCs.

\subsubsection{Significantly Higher Levels of IFN- $\gamma$ and IL-4 Induced by Recombinant Strains but Significantly Lower IFN- $\alpha$ than Control} As summarized in (Figure 3 ) in the sera of mice the concentrations mean of anti viral cytokine (IFN- $\alpha$ ), T regulatory cell cytokines (IL-10 and TGF- $\beta$ ), proinflammatory cytokines (IL-12), Th2 cytokine (IL-4), and Th1 cytokine (IFN- $\gamma$ ). There were higher concentrations of IL- 4 and IFN- $\gamma$ in the $L$. plantaram NC8-pSIP409-pgsA-Vp6-DCpep or L. plantaram NC8-pSIP409-pgsA-Vp7-DCpep group than the control groups, signifying an effect of adjuvant in LAB on both responses of Th1 and Th2 type (Figure 3). There were 4 to 11 fold higher concentrations mean of IL-4 and IFN- $\gamma$ in the $L$. plantaram NC8-pSIP409-pgsAVP6-DCpep or L. plantaram NC8-pSIP409-pgsA-VP7-DCpep group contrasted to the control group.

\subsubsection{Concentrations of Serum IFN- $\alpha$ Significantly Correlated with}

TLR3- and TLR9-Expressing CD14- APCs Frequencies in Spleen

To recognize the associations among frequencies of TLR2, TLR3 and TLR9 expressing APCs in spleen or blood and serum concentrations of the cytokines between the four treatment groups the Spearman's rank correlation analysis was performed. Significant positive correlations were found between concentrations of IFN- $\alpha$ and frequencies of TLR3 expressing CD14- cDC and macrophages/monocytes and TLR9 expressing CD14- CDC and macrophages/monocytes in spleen. These associations recommend that the CD14- APCs in spleen may significantly contribute to the levels of IFN- $\alpha$ in serum.

\section{Discussion}

In this study, we evaluated the frequencies of systemic TLR2, TLR3 and TLR9 expressing CD14+ and CD14- APCs in mice colonized with L. plantaram NC8, immunized with L. plantaram NC8-pSIP409-pgsA-Vp6-DCpep or L. plantaram NC8-pSIP409-pgsA-Vp7-DCpep. The expression pattern was evaluated by anti 
porcine TLR antibodies at the intracellular TLR expression in the defined mice APC and protein level for cell surface.

On early postnatal growth of the systemic innate immune cells the L. plantaram intestinal colonization alone had a profound effect on stimulating, as confirmed by the significantly augmented frequencies of macrophages/monocytes and cDCs in spleen [17] [18] [19] [20] [21], theTLR2 and TLR9 expressing CD14+ cDCs in blood increased significantly, and the serum cytokine IL-12 level elevated significantly. The IL-12 responses and TLR2 and TLR9 expressing CDC induction can be elucidated, as a minimum somewhat, by the availability of TLR2 and TLR9 ligands in the LAB. The TLR2 and TLR9 expressing CDC induction and the IFN- $\gamma$ and IL-4 enhancement of responses may provide the mechanism for the adjuvanticity of LAB in enhancing humoral and cellular immune responses stimulated by rotavirus vaccines, influenza virus, poliovirus and or Salmonella typhi Ty21a infections [18] [20] [21] [26] [27].

An additive effect of $L$. plantaram NC8-pSIP409-pgsA-Vp6-DCpep or $L$. plantaram NC8-pSIP409-pgsA-Vp7-DCpep in TLR2 and TLR9 expressing CD14+ APCs activation in spleen and CD14- CDCs in blood was observed. In contrast, the frequencies increased of TLR2 and TLR9 expressing APCs in $L$. plantaram NC8-pSIP409-pgsA-Vp6-DCpep or L. plantaram NC8-pSIP409-pgsAVp7-DCpepmice simply may be because of the significantly higher counts of $L$. plantaram NC8, which may perhaps interpret to an augmented amount of TLR agonists obtainable to the host immune system stimulation. on the other hand, due to the total frequencies of APCs or CD14+ APCs in the L. plantaram NC8-pSIP409-pgsA-Vp6-DCpep or L. plantaram NC8-pSIP409-pgsA-Vp7-DCpepmice similar from the NC8-pSIP409-pgsA mice in blood, and the APCs total in spleen of the L. plantaram NC8-pSIP409-pgsA-Vp6-DCpep or L. plantaram NC8-pSIP409-pgsA-Vp7-DCpepmice were significantly lower than the L. plantaram NC8only group, it is not possible that the higher L. plantaram NC8 count in the L. plantaram NC8-pSIP409-pgsA-Vp6-DCpep or L. plantaram NC8pSIP409-pgsA-Vp7-DCpepgroup played a further relevant function in the increases of TLR2 and TLR9 expressing APC frequencies than of NC8-pSIP409pgsA.

The CD14+ and CD14- APCs expressing TLR2, TLR3 and TLR9 were both stimulated in spleen by PRV infection; on the other hand, the responses in blood of TLR expressing APC were incredibly dissimilar from that of spleen. In blood of the NC8-pSIP409-pgsA mice the frequencies of TLR2, TLR3 and TLR9 expressing APCs were comparable to the control PBS mice. In the NC8pSIP409-pgsA mice group the lack of TLR responses in blood is the same with previous results showing that $\mathrm{PRV}$ infection did not augment total frequencies of APCs or CD14+ cDCs, and in blood reduced significantly the frequencies total of CD14+ macrophages/monocytes [17] [18] [19] [20] [21]. The significant dissimilarity among the APC response in blood and spleen the probable causes may be due to MNCs were only at one time point collected. Our study gives ad- 
ditional confirmation that TLR9 is involved in induction of the innate immune responses by both recombinant $L$. plantaram NC8-pSIP409-pgsA-Vp6-DCpep or L. plantaram NC8-pSIP409-pgsA-Vp7-DCpep because the highest frequencies induced of TLR9 expressing CD14+ macrophages/monocytes in blood and spleen, which is the same results with the effect of DCpep adjuvant vaccine B and $\mathrm{T}$ cell immune responses induced [27]. In spleen and blood the IFN- $\alpha$ level reduction associated significantly with the frequencies reduction of TLR3 and TLR9 expressing CD14- APCs, which perhaps recommend that the action of CD14- APCs significantly contributes to the response of IFN- $\alpha$. The $L$. plantaram NC8 on IFN- $\gamma$, IL-4, IFN- $\alpha$ and TGF- $\beta$ serum responses had significant influence.

\section{Conclusion}

This study after $L$. plantaram NC8 colonization elucidated the systemic responses of TLR2, TLR3, and TLR9 expressing macrophage/and monocyte CDC, by recombinant $L$. plantaram NC8-pSIP409-pgsA-Vp6-DCpep or L. plantaram NC8-pSIP409-pgsA-Vp7-DCpep. Our results help the sympathetic of the mechanism for LAB's adjuvant effect on rotavirus vaccines and the different induction of adaptive and innate immune responses by $L$. plantaram NC8 colonization against rotavirus infection.

\section{Declarations}

\section{Ethics Approval and Consent to Participate}

This study was carried out in agreement with the principles established by Jilin Agriculture University Changchun China and guide for the use of laboratory and care animals and all experimental protocols were approved by a Jilin Agriculture University (No. JLAU08201007).

\section{Consent for Publication}

Not applicable. Availability of data and materials: will be provided after publication when required.

\section{Funding}

This work was supported by The National Key Research and Development Program of China (2017YFD0501000).

\section{Authors' Contributions}

Author 1 SMS-searching data, wrote manuscript and acted as corresponding author. Author 2 WY-editing manuscript. Author 3 GY-editing the manuscript and supervision of the manuscript. Author $4 \mathrm{CW}$-editing the manuscript and supervision of the manuscript.

\section{Acknowledgements}

We are thankful to the Jilin Agricultural University for the internet to download 
Data for this study.

\section{Conflicts of Interest}

The authors declare no conflicts of interest regarding the publication of this paper.

\section{References}

[1] Wen, K., Azevedo, M.S., Gonzalez, A., Zhang, W., Saif, L.J., Li, G., Yousef, A. and Yuan, L. (2009) Toll-Like Receptor and Innate Cytokine Responses Induced by Lactobacilli Colonization and Human Rotavirus Infection in Gnotobiotic Pigs. Veterinary Immunology and Immunopathology, 127, 304-315. https://doi.org/10.1016/j.vetimm.2008.10.322

[2] Kaisho, T. and Akira, S. (2006) Toll-Like Receptor Function and Signaling. Journal of Allergy and Clinical Immunology, 117, 979-987. https://doi.org/10.1016/j.jaci.2006.02.023

[3] Akira, S. and Hemmi, H. (2003) Recognition of Pathogen-Associated Molecular Patterns by TLR Family. Immunology Letters, 85, 85-95. https://doi.org/10.1016/S0165-2478(02)00228-6

[4] Uematsu, S. and Akira, S. (2006) Toll-Like Receptors and Innate Immunity. Journal of Molecular Medicine, 84, 712-725. https://doi.org/10.1007/s00109-006-0084-y

[5] Seth, R.B., Sun, L., Ea, C.K. and Chen, Z.J. (2005) Identification and Characterization of MAVS, a Mitochondrial Antiviral Signaling Protein that Activates NF- $\kappa \mathrm{B}$ and IRF 3. Cell, 122, 669-682. https://doi.org/10.1016/j.cell.2005.08.012

[6] Sandor, F. and Buc, M. (2005) Toll-Like Receptors. II. Distribution and Pathways Involved in TLR Signalling. Folia Biologica, 51, 188-197.

[7] Hug, H., Mohajeri, M.H. and La Fata, G. (2018) Toll-Like Receptors: Regulators of the Immune Response in the Human Gut. Nutrients, 10, 203. https://doi.org/10.3390/nu10020203

[8] Sieling, P.A. and Modlin, R.L. (2002) Toll-Like Receptors: Mammalian "Taste Receptors" for a Smorgasbord of Microbial Invaders. Current Opinion in Microbiolo$g y$, 5, 70-75. https://doi.org/10.1016/S1369-5274(02)00288-6

[9] Parashar, U.D., Gibson, C.J., Bresee, J.S. and Glass, R.I. (2006) Rotavirus and Severe Childhood Diarrhea. Emerging Infectious Diseases, 12, 304-306.

https://doi.org/10.3201/eid1202.050006

[10] Shonyela, S.M., Wang, G., Yang, W., Yang, G. and Wang, C. (2017) New Progress regarding the Use of Lactic Acid Bacteria as Live Delivery Vectors, Treatment of Diseases and Induction of Immune Responses in Different Host Species Focusing on Lactobacillus Species. World Journal of Vaccines, 7, 43-75. https://doi.org/10.4236/wjv.2017.74004

[11] Ljungh, A. and Wadstrom, T. (2006) Lactic Acid Bacteria as Probiotics. Current Issues in Intestinal Microbiology, 7, 73-89.

[12] Herias, M.V., Hessle, C., Telemo, E., Midtvedt, T., Hanson, L.A. and Wold, A.E. (1999) Immunomodulatory Effects of Lactobacillus plantarum Colonizing the Intestine of Gnotobiotic Rats. Clinical and Experimental Immunology, 116, 283-290. https://doi.org/10.1046/j.1365-2249.1999.00891.x

[13] Yasui, H., Shida, K., Matsuzaki, T. and Yokokura, T. (1999) Immunomodulatory Function of Lactic Acid Bacteria. Antonie van Leeuwenhoek, 76, 383-389. 
https://doi.org/10.1023/A:1002041616085

[14] Zocco, M.A., dal Verme, L.Z., Cremonini, F., Piscaglia, A.C., Nista, E.C., Candelli, M., Novi, M., Rigante, D., Cazzato, I.A., Ojetti, V., et al. (2006) Efficacy of Lactobacillus GG in Maintaining Remission of Ulcerative Colitis. Alimentary Pharmacology \& Therapeutics, 23, 1567-1574. https://doi.org/10.1111/j.1365-2036.2006.02927.x

[15] Shornikova, A.V., Casas, I.A., Isolauri, E., Mykkanen, H. and Vesikari, T. (1997) Lactobacillus Reuteri as a Therapeutic Agent in Acute Diarrhea in Young Children. Journal of Pediatric Gastroenterology and Nutrition, 24, 399-404. https://doi.org/10.1097/00005176-199704000-00008

[16] Majamaa, H., Isolauri, E., Saxelin, M. and Vesikari, T. (1995) Lactic Acid Bacteria in the Treatment of Acute Rotavirus Gastroenteritis. Journal of Pediatric Gastroenterology and Nutrition, 20, 333-338. https://doi.org/10.1097/00005176-199504000-00012

[17] Zhang, W, Azevedo, M.S.P., Gonzalez, A.M., Saif, L.J., Van Nguyen, T., Wen, K., Yousef, A.E. and Yuan, L. (2008) Influence of Probiotic Lactobacilli Colonization on Neonatal B cell Responses in a Gnotobiotic Pig Model of Human Rotavirus Infection and Disease. Veterinary Immunology and Immunopathology, 122, 175-181. https://doi.org/10.1016/j.vetimm.2007.10.003

[18] Link-Amster, H., Rochat, F., Saudan, K.Y., Mignot, O. and Aeschlimann, J.M. (1994) Modulation of a Specific Humoral Immune Response and Changes in Intestinal Flora Mediated through Fermented Milk Intake. FEMS Immunology and Medical Microbiology, 10, 55-63. https://doi.org/10.1111/j.1574-695X.1994.tb00011.x

[19] Olivares, M., Diaz-Ropero, M.P., Sierra, S., Lara-Villoslada, F., Fonolla, J., Navas, M., Rodriguez, J.M. and Xaus, J. (2007) Oral Intake of Lactobacillus fermentum CECT5716 Enhances the Effects of Influenza Vaccination. Nutrition, 23, 254-260. https://doi.org/10.1016/j.nut.2007.01.004

[20] de Vrese, M., Rautenberg, P., Laue, C., Koopmans, M., Herremans, T. and Schrezenmeir, J. (2005) Probiotic Bacteria Stimulate Virus-Specific Neutralizing Antibodies Following a Booster Polio Vaccination. European Journal of Nutrition, 44, 406-413. https://doi.org/10.1007/s00394-004-0541-8

[21] Kaila, M., Isolauri, E., Soppi, E., Virtanen, E., Laine, S. and Arvilommi, H. (1992) Enhancement of the Circulating Antibody Secreting Cell Response in Human Diarrhea by a Human Lactobacillus Strain. Pediatric Research, 32, 141-144. https://doi.org/10.1203/00006450-199208000-00002

[22] Miura, K., Ishioka, M. and Iijima, K. (2017) The Roles of the Gut Microbiota and Toll-like Receptors in Obesity and Nonalcoholic Fatty Liver Disease. Journal of $O b$ esity \& Metabolic Syndrome, 26, 86-96. https://doi.org/10.7570/jomes.2017.26.2.86

[23] Zhu, J., Yang, Q., Cao, L., Dou, X., Zhao, J., Zhu, W., Ding, F., Bu, R.-E., Suo, S., Ren, Y., et al. (2013) Development of Porcine Rotavirus vp6 Protein Based ELISA for Differentiation of This Virus and Other Viruses. Virology Journal, 10, 91. https://doi.org/10.1186/1743-422X-10-91

[24] Azevedo, M.S.P., Yuan, L., Pouly, S., Gonzales, A.M., Jeong, K.I., Nguyen, T.V. and Saif, L.J. (2006) Cytokine Responses in Gnotobiotic Pigs after Infection with Virulent or Attenuated Human Rotavirus. Journal of Virology, 80, 372-382. https://doi.org/10.1128/JVI.80.1.372-382.2006

[25] Wen, K., Azevedo, M.S.P., Gonzalez, A., Zhang, W., Saif, L.J., Li, G., Yousef, A. and Yuan, L. (2009) Toll-Like Receptor and Innate Cytokine Responses Induced by Lactobacilli Colonization and Human Rotavirus Infection in Gnotobiotic Pigs. Ve- 
terinary Immunology and Immunopathology, 127, 304-315.

https://doi.org/10.1016/j.vetimm.2008.10.322

[26] Isolauri, E., Joensuu, J., Suomalainen, H., Luomala, M. and Vesikari, T. (1995) Improved Immunogenicity of Oral D x RRV Reassortant Rotavirus Vaccine by Lactobacillus casei GG. Vaccine, 13, 310-312.

https://doi.org/10.1016/0264-410X(95)93319-5

[27] Zhang, W., Azevedo, M.S., Wen, K., Gonzalez, A., Saif, L.J., Li, G., Yousef, A.E. and Yuan, L. (2008) Probiotic Lactobacillus Acidophilus Enhances the Immunogenicity of an Oral Rotavirus Vaccine in Gnotobiotic Pigs. Vaccine, 26, 3655-3661. https://doi.org/10.1016/j.vaccine.2008.04.070

\section{Abbreviations and Acronynys}

$\begin{array}{ll}\text { BALB/c } & \text { Albino laboratory bred strain of house mouse } \\ \text { CD } & \text { Cluster of differentiation } \\ \text { DCpep } & \text { Dendritic cell targeting peptide } \\ \text { IgG } & \text { Immunogloblin G } \\ \text { L. } & \text { Lactobacillus } \\ \text { PP } & \text { Peyer's patch } \\ \text { PRV } & \text { Porcine rotavirus } \\ \text { pgsA } & \text { Poly- } \gamma \text {-glutamic acid synthetase A } \\ \text { sIgA } & \text { Immunoglobulin A } \\ \text { PRV } & \text { Porcine rotavirus } \\ \text { TLR } & \text { Toll-like receptors } \\ \text { IFN- } \gamma & \text { Interferon gamma } \\ \text { IFN- } \alpha & \text { Interferon alpha }\end{array}$

